\title{
Crop Advisor: A Software Tool for Forecasting Paddy Yield
}

\author{
Dr.S. Veenadhari
}

\begin{abstract}
The highly erratic rainfall and associated climatic parameters in India, have greater influence on the performance of cropping systems and are adversely affecting the crop yields. Forecasting of crop yields from the climatic input parameters would not only help the farmers in deciding the sale price of their produce, but it also helps the policy makers to decide the minimum support price and the buffer stock expected. Such predictions will also help the associated industries for planning the logistics of their business. Several methods of predicting and modeling crop yields have been developed in the past with varying rates of success. Statistical models often don't take into account characteristics of the weather, they are mostly are empirical in nature. In the present study a software tool named 'Crop Advisor' has been developed as a user friendly web page. This website is designed as an interactive software tool for predicting the influence of climatic parameters on the crop yields. C 4.5 algorithm is used to find out the most influencing climatic parameter on the crop yields of selected crops in selected districts of Madhya Pradesh. This software provides an indication of relative influence of different climate parameters on the crop yield, other agro-input parameters responsible for crop yield are not considered in this tool, since, application of these input parameters varies with individual fields in space and time.
\end{abstract}

\section{INTRODUCTION}

$\mathrm{C}$ ROP production is a complex phenomenon that is influenced by agro-climatic input parameters. Agriculture input parameters varies from field to field and farmer to farmer. Collecting such information on a larger area is a daunting task. However, the climatic information collected in India at every 1 sq.m area in different parts of the district is tabulated by Indian Meteorological Department. The huge such data sets can be used for predicting their influence on major crops of that particular district or place. There are different forecasting methodologies developed and evaluated by the researchers all over the world in the field of agriculture or associated sciences. Some of the such studies are: Studies conducted by agricultural researchers in Pakistan have shown that attempts of crop yield maximization through pro-pesticide state policies have led to a dangerously high pesticide usage. These studies have reported negative correlation between pesticide usage and crop yield[1]. In their study they have shown that how data mining integrated agricultural data including pest scouting, pesticide usage and meteorological recordings are useful for optimization of pesticide usage.

Dr.S. Veenadhari, Associate Professor, AISECT University. E-mail:veenadhari1@gmail.com DOI: 10.9756/BIJDM.10461
Thematic information related to agriculture which has spatial attributes was reported in one of the study [6]. Their study aimed at discerning trends in agriculture production with references to the availability of inputs. K-means method was used to perform forecasts of the pollution in the atmosphere [4], the $\mathrm{k}$ nearest neighbor was applied for simulating daily precipitations and other weather variables [11], and different possible changes of the weather scenarios are analyzed using SVMs [13]. Data mining techniques are often used to study soil characteristics. As an example, the k-means approach is used for classifying soils in combination with GPS-based technologies [14]. Apples were checked using different approaches before sending them to the market. [9], uses a kmeans approach to analyze color images of fruits as they run on conveyor belts[12].Uses X-ray images of apples to monitor the presence of watercores, and a neural network is trained for discriminating between good and bad apples. Spatial data mining introduced especially decision tree algorithm applying to agriculture land grading [15]. He combined spatial data mining/decision tree techniques with expert system techniques and applied them to establish an intelligent agriculture land grading information system. The author adopted decision tree c4.5 algorithm and implement with Mo2.0 and $\mathrm{VC}++6.0$ to build agriculture land grading expert system. The study showed the particular advantages of this methodology in addressing problems in land grading such as missing land information, difficulties in quantitative analysis of factors. A decision tree classifier for agriculture data was proposed [5]. Although some data set includes missing values, the experiment showed the performance of the proposed method. This new classifier uses new data expression and can deal with both complete data and incomplete data. In the experiment, 10 -fold cross validation method is used to test abalone data set, horse-colic data set and soybean data set. Their results showed the proposed decision tree is capable of classifying all kinds of agriculture data. Data mining techniques for evolution of association rules for droughts and floods in India was applied using climate inputs [2].In their study, a data-mining algorithm using the concepts of minimal occurrences with constraints and time lags was used to discover association rules between extreme rainfall events and climatic indices. Rainfall events were forecasted the using data mining techniques [7].The occurrence of prolonged dry period or heavy rain at the critical stages of the crop growth and development may lead to significant reduction in crop yield. Sugarcane yield estimated at municipality level in SãoPaulo State, Brazil, using 10-day periods of SPOT Vegetation NDVI images and ECMWF meteorologicaldata [3].Data Mining Approach based on Spatio-Temporal data to forecast irrigation water demand [8]. In their study data set were prepared containing information on suitable attributes obtained from 
three different sources namely meteorological data, remote sensing images and water delivery statements. In order to make the prepared data sets useful for demand forecasting and pattern extraction data sets were processed using a novel approach based on a combination of irrigation and data mining knowledge. Decision tree techniques were applied to forecast future water requirement.

\section{MethodOLOGY}

The present study was aimed to develop a web site for finding out the influence of climatic parameters on crop production in selected districts of Madhya Pradesh. The selection of districts has been made based on the area under that particular crop. Based on this criteria first top five districts in which the selected crop area is maximum were selected. The crops selected in the study is based on the predominate crops in the selected district. The selected crop includes: Soybean, Maize, Paddy and Wheat. The yield of these crops was tabulated for continuous 20 years by collecting the information from secondary sources. Similarly for the corresponding years climatic parameters such as Rainfall, Maximum temperature, Minimum temperature, Potential Evapotranspiration, Cloud cover, Wet day frequency were also collected from the secondary sources. For analysis in developing the software .net(c\#) is used as a front end tool, and SQL server 2008 is used as back end tool to maintain the database. C4.5 algorithm was used in the analysis. C4.5 is an algorithm which is a successor to ID3 (Iterative Dichotomies 3 ) is used in study [10]. It is also based on Hunt's algorithm. C4.5 handles both categorical and continuous attributes to build a decision tree and in this study the collected input parameters are continuous values. In order to handle continuous attributes, C4.5 splits the attribute values into two partitions based on the selected threshold such that all the values above the threshold as one child and the remaining as another child. It also handles missing attribute values. In pseudo code the algorithm is:

1. Check for base cases

2. For each attribute ' $a$ ' (Find the normalized information gain from splitting on a)

3. Let ' $a$ ' best be the attribute with the highest normalized information gain

4. Create a decision node that splits on 'a' best

5. Recur on the sub lists obtained by splitting on a best, and add those nodes as children of node.

\section{Entropy and Gain Calculations}

Let $\mathrm{S}$ be a set consisting of $\mathrm{s}$ data samples. Suppose the class label attribute has $\mathrm{m}$ distinct values defining $\mathrm{m}$ distinct classes, $C_{i}$ (for $\left.i=1, \ldots, m\right)$. Let $s_{i}$ be the number of samples of $S$ in class $\mathrm{C}_{\mathrm{i}}$. The expected information needed to classify a given sample is given by:

$$
I\left(s_{1}, s_{2}, \ldots, s_{m}\right)=-\sum_{i=1}^{m} p_{i} \log _{2}\left(p_{i}\right)
$$

Where pi is the probability that an arbitrary sample belongs to class $\mathrm{Ci}$ and is estimated by si/s. Note that log function to the base 2 is used since the information is encoded in bits.

Let attribute $A$ have $v$ distinct values $\left\{a_{1}, a_{2}, \ldots a_{v}\right\}$. Attribute $A$ can be used to partition $S$ into v subsets, $\left\{S_{1}, S_{2}\right.$, $\left.\ldots, S_{v}\right\}$, where $\mathrm{Sj}$ contains those samples in $\mathrm{S}$ that have values $a_{j}$ of A. If A were selected as the test attribute ( i.e., the best attribute for splitting), then these subsets would correspond to the branches grown from the node containing the set $\mathrm{S}$. Let $\mathrm{s}_{\mathrm{ij}}$ be the number of samples of class $C_{i}$ in a subset $S_{j}$. The entropy, or expected information based on the partitioning into subsets by $A$, is given by the subset (i.e., having value $a_{j}$ of $A$ ) divided by the total number of samples in S. The smaller the entropy value, the greater the purity of the subset partitions. Note that for a given subset $S_{j}$

$$
I\left(s_{1 j}, s_{2 j}, \ldots, s_{m j}\right) \underset{i=1}{m} \sum p_{i j} \log _{2}\left(p_{i j}\right)
$$

Where and is the probability that a sample in $\mathrm{S}_{\mathrm{j}}$ belongs to class $\mathrm{C}_{\mathrm{i}}$. Encoding the information that would be gained by branching on $\mathrm{A}$ is

$$
\text { Gain }(A)=I\left(s_{1}, s_{2}, \ldots, s_{m}\right)-E(A)
$$

In other words, Gain $(A)$ is the expected reduction in entropy caused by knowing the value of attribute A. Decision tree is constructed based on the maximum gain value till there are no remaining attributes on which the tuples may be further partitioned.

\section{RESULTS AND DISCUSSION}

The collected information of climate and crop yield data were tabulated and average values of each of these parameters for continuous 20 years were worked out. To represent the calculations used in the study a sample table for paddy crop in Balaghat district of Madhya Pradesh is presented in the following table 1 . 


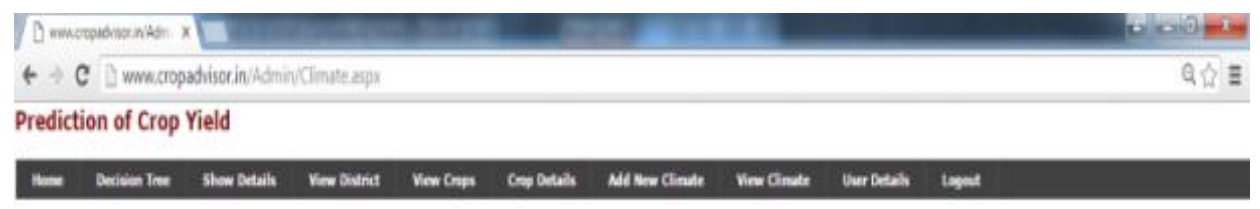

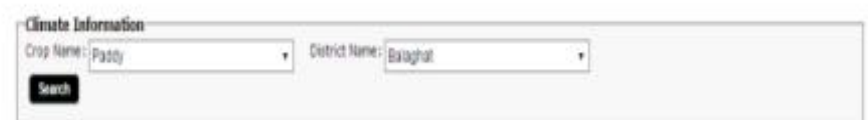

\section{때요}

\begin{tabular}{|c|c|c|c|c|c|c|c|c|c|}
\hline $5 . \mathrm{No}$ & Rais fill & $\operatorname{Max} T=0$ & Me Teng & ตี & Wetay & Clood Cover & Viett & Estit & Daldte \\
\hline 1 & 144394 & 11,100 & 24.72 & 6510 & 420 & 18960 & 1166.000 & 1 & 0 \\
\hline 2 & 2813010 & 12.960 & $21.6 \%$ & 6580 & 489 & 34.60 & Late.to0 & I & 0 \\
\hline 1 & 1655 & 12.180 & 21.126 & 6500 & 4,320 & 18.786 & म2actow & 1 & 0 \\
\hline 4 & 100MSit & 11.480 & 2190 & $6 \in 10$ & 6.670 & 18.90 & 144.600 & ? & 0 \\
\hline 3 & 128967 & 12770 & 20.126 & 60000 & 4930 & 00.100 & 1257.000 & ? & 0 \\
\hline 6 & Haxato & 12.930 & 2450 & 6.60 & 5.120 & 48500 & 1257:200 & l & 0 \\
\hline 7 & In2:160 & 12.900 & 2130 & 6040 & $5: 200$ & 42100 & Hiss.tow & l & 0 \\
\hline 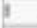 & 108510 & 38.40 & 2.30 & 6500 & 460 & $36 \%$ & Hete.00 & l & 0 \\
\hline 9 & 1231240 & s.:210 & $20 \mathrm{~s} 20$ & 6050 & 6960 & 40600 & 1550.000 & 7 & 0 \\
\hline II & Het.3: & 13.670 & 2195 & 650 & 470 & 38.70 & IIUED & I & 0 \\
\hline II & 1567:000 & $12: 230$ & 20.20 & 6500 & 6.760 & 40,600 & 1aritovo & ? & 0 \\
\hline 12 & LWDSO & 13.250 & 2107 & 6.540 & 5.140 & 18.130 & 1555100 & l? & 0 \\
\hline 17 & 181000 & 13900 & 21.50 & 650 & 6.660 & 28.150 & 106:000 & ? & 0 \\
\hline 4 & 1175 Doto & 133.250 & 20.500 & 6540 & 4650 & $25: 200$ & 1146.600 & 1 & 0 \\
\hline 15 & 10020018 & satso & zasto & 6.220 & 4.00 & 60.19 & แm2000 & ? & 0 \\
\hline 16 & 1535098 & 32.570 & $20: 60$ & 6.620 & 4.150 & 4.120 & 2057.000 & l & 0 \\
\hline 17 & 124:ast & 18510 & $20.5 \mathrm{tt}$ & (4) & 9,400 & 19960 & Hs:.00 & ? & 0 \\
\hline u & 1584000 & 32.560 & 22040 & 6550 & 4.520 & 30.562 & Laraccos & 12 & 0 \\
\hline 98 & 126t.95t & 18.560 & $29 a b$ & 652 & 4.660 & 60.650 & bet.000 & ! & 0 \\
\hline 20 & 1122020 & 12.aso & 22540 & 6.500 & $5: 100$ & 40.000 & ตุละด & ? & 0 \\
\hline
\end{tabular}

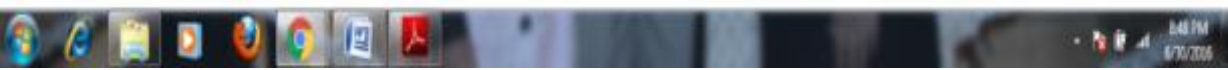

Using such tabulated values a software was developed in home page contains, the methodology adopted in the study .net as a front end tool and sql server as back end support. The software is further developed into user friendly web site. The and registration of the new users. The screen shot of the home page of the website is presented below.

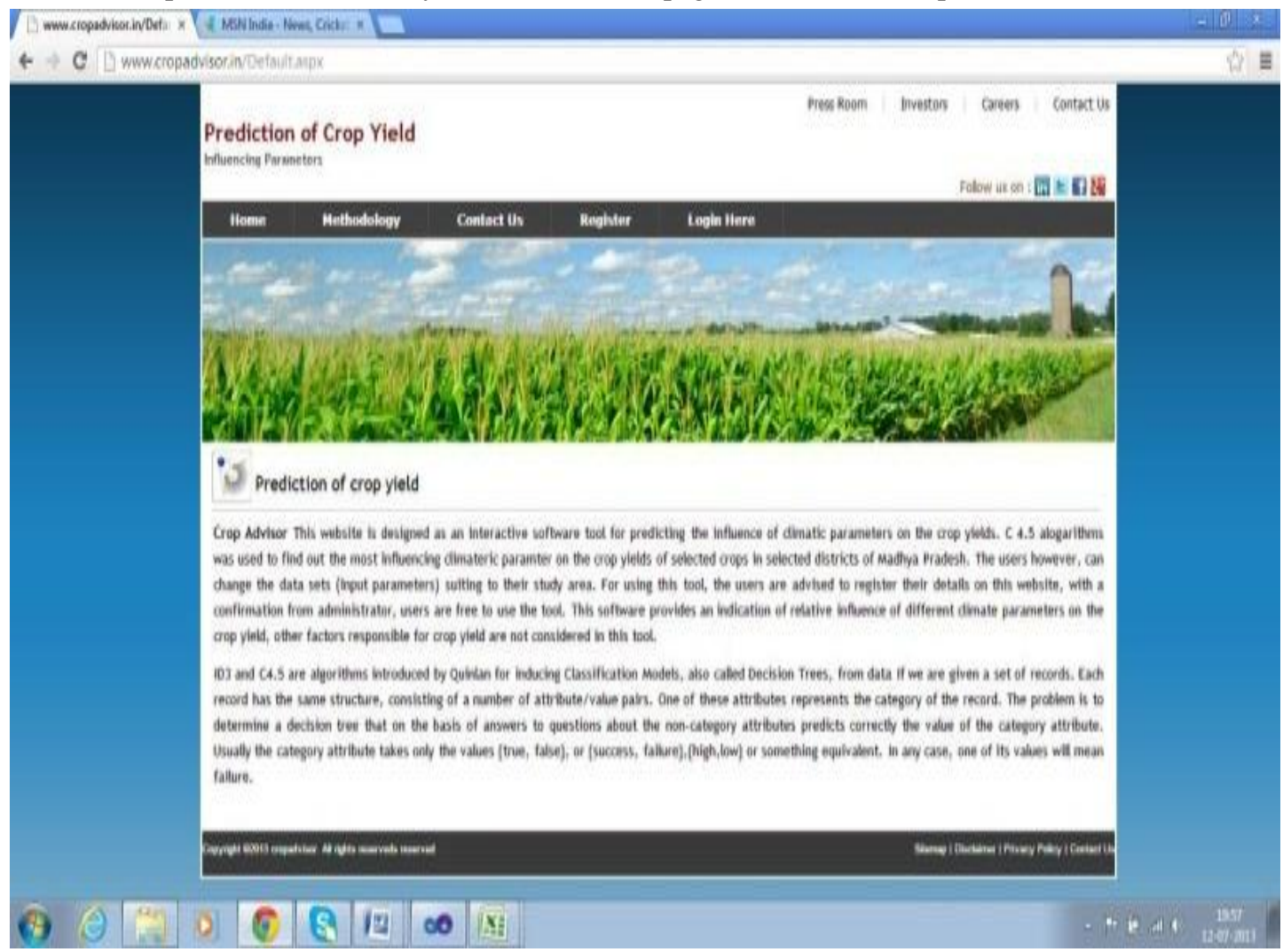


The first time users of the website need the administrators approval for login into the site for further use. Once the user gives the login and password details the next screen appears as:

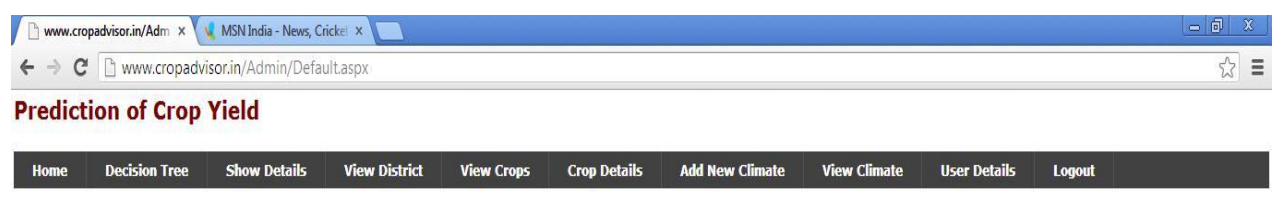

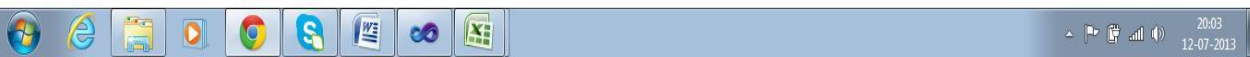

From the tabulated data, decision tree is constructed using c4.5 algorithm based on gain values of each attribute. After calculating the gain, the attribute with the highest gain value is selected as root node and is further splits the table into tree, this process continues recursively till all the tuples belong to the same class. The screen shot of the decision tree constructed is presented below:

Prediction of Crop Yield

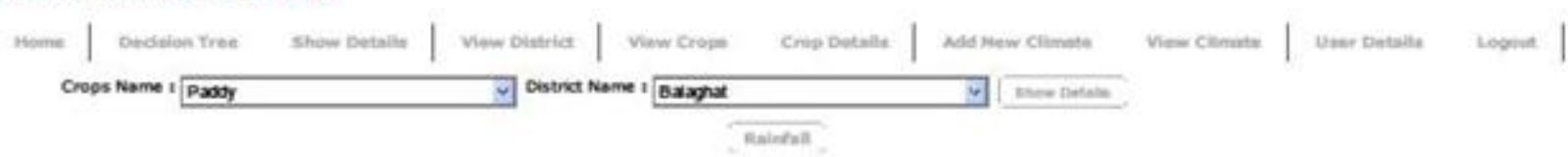

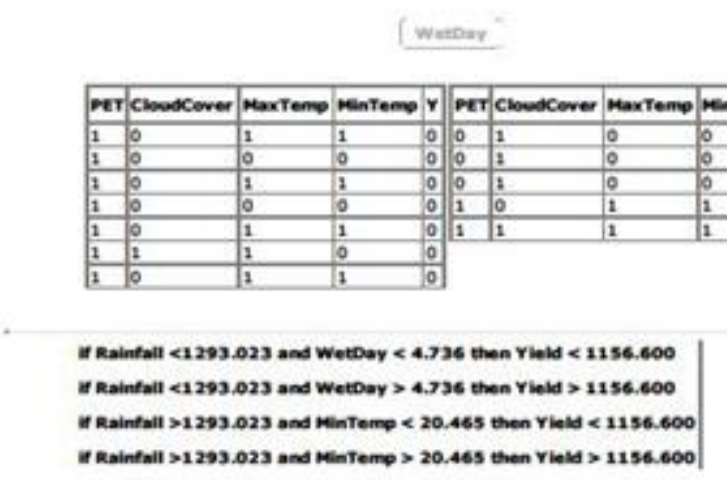

In the above screen shot it was revealed that paddy crop yield is influenced mostly by the climatic parameter Rainfall, followed by wetday and minimum temperature. The decision rules thus formed for forecasting yield as follows.

1. if Rainfall $<1293.023$ and WetDay $<4.736$ then Yield $<1156.600$

2. if Rainfall <1293.023 and WetDay > 4.736 then Yield $>1156.600$

3. if Rainfall $>1293.023$ and MinTemp $<20.465$ then
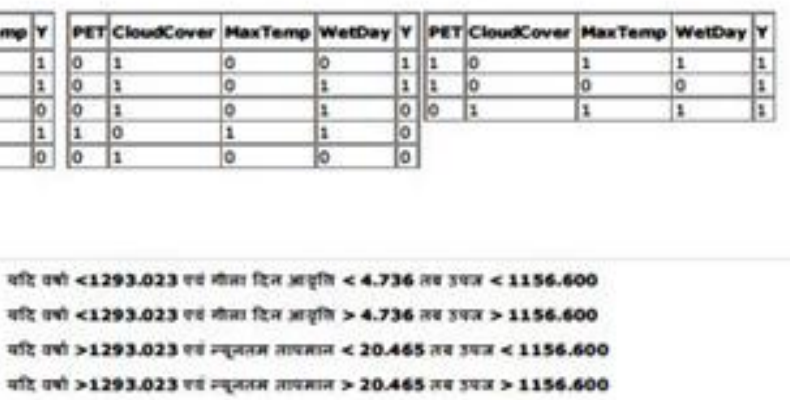


\section{ACKNOWLEDGEMENT}

The first author would like to extend her heartfelt gratitude to Vice Chancellor, MGCGV, Chitrakoot for giving admission to pursue Doctoral program from the university. Thanks are also due to Director, CIAE, Bhopal for extending the facilities to carry out the research activities in the Institute. All the help received from the staff of the University and the Institute is duly acknowledged.

\section{REFERENCES}

[1] A. Abdullah, S. Brobst, I. Pervaiz, M. Umer and A. Nisar, "Learning dynamics of pesticide abuse through data mining", Proceedings of Australian Workshop on Data mining and Web Intelligence, New Zealand, January, 2004.

[2] C.T. Dhanya and D. Nagesh Kumar, "Data mining for evolution of association rules for droughts and floods in India using climate inputs", J. of Geo. Phy. Res.114: 1-15, 2009.

[3] F.L. Fernandes, Jansle Vieira Rocha, Rubens Augusto and Camargo Lamparelli, "Sugarcane yield estimates using time series analysis of spot vegetation images", Sci. Agric. (Piracicaba, Braz.), Vol. 68, No.2, 2011.

[4] H. Jorquera, R. Perez, A. Cipriano and G. Acuna, "Short term forecasting of air pollution episodes", Zannetti P (eds) Environmental Modeling, WIT Press, UK, 2001.

[5] Jun Wu, Anastasiya Olesnikova, Chi-Hwa Song and Won Don Lee, "The Development and Application of Decision Tree for Agriculture Data", IITSI, Pp.16-20, 2009.

[6] C. Kiran Mai, I.V. Murali Krishna and A. Venugopal Reddy, "Data Mining Of Geospatial Database For Agriculture Related Application", Proceedings of Map India, 2006.

[7] M. Kannan, S. Prabhakaran and P. Ramachandran, "Rainfall forecasting using data mining technique", International Journal of Engineering and Technology, Vol.2, No.6, Pp.397-401, 2010.

[8] A. Khan Mohammad, Md. Zahidul Islam and Mohsin Hafeez, "Evaluating the Performance of Several Data Mining Methods for Predicting Irrigation Water Requirement", Proceedings of the Tenth Australasian Data Mining Conference (AusDM 2012), Pp.199-207, 2012.

[9] V. Leemans and M.F. Destain, "A real-time grading method of apples based on features extracted from defects", J. Food Eng. 61, Pp.83-89, 2004.

[10] J.R. Quilan, Decision trees and multi-valued attributes, Oxford University Press, Inc, 1988.

[11] B. Rajagopalan and U. Lall, "A k-nearest-neigmulator for daily precipitation and other weather variables", Wat REs Res, Vol.35, No.10, Pp.3089-3101, 1999.

[12] M.A. Shahin, E.W. Tollner, R.W. McClendon and H.R. Arabnia, “Apple classification based on surface bruises using image processing and neural networks", Trans. ASAE 45, Pp.1619- 1627, 2002.

[13] S. Tripathi, V.V. Srinivas and R.S. Nanjudiah, "Downscaling of precipitation for climate change scenarios: a support vector machine approach", J. Hydrol, Vol.330, Pp.621- 640, 2006.

[14] K. Verheyen, Adrianens, M. Hermy and S. Deckers, "High resolution continuous soil classification using morphological soil profile descriptions", Geoderma, Vol.101, Pp.31-48, 2001.

[15] Zelu Zia, "An Expert System Based on Spatial Data Mining Used Decision Tree for Agriculture Land Grading", Second International Conference on Intelligent Computation Technology and Automation, 2009. 